\title{
Global Governance als Polycentric Governance
}

\author{
Benjamin Faude (LSE)
}

\begin{abstract}
In seiner „Theory of Global Governance“ argumentiert Michael Zürn, Staaten seien gegenwärtig in ein globales politisches System eingebettet, das eine Vielzahl internationaler Institutionen umfasst. Dieser Forumsbeitrag unternimmt den Versuch einer Annäherung an die Gestalt des von Zürn identifizierten globalen politischen Systems. Er schlägt vor, Global Governance als ein polyzentrisch strukturiertes System zu konzeptualisieren, das aus vielen Entscheidungszentren besteht, die formal voneinander unabhängig sind, sich aber faktisch wechselseitig beeinflussen. Der Beitrag verdeutlicht zunächst, worin die zentralen Merkmale der polyzentrischen Struktur des von Zürn identifizierten globalen politischen Systems bestehen. Anschließend skizziert er, wie die polyzentrische Struktur staatliches Verhalten beeinflusst und das Problem politischer Ordnungsbildung jenseits des Nationalstaates akzentuiert. Abschließend wird vor dem Hintergrund gegenwärtiger Herausforderungen für zwischenstaatliche Kooperation angedeutet, worin die institutionellen Stärken einer polyzentrischen Regelungsstruktur liegen.
\end{abstract}

\section{Einleitung ${ }^{1}$}

In seiner „Theory of Global Governance“ entfaltet Michael Zürn das Argument, innerhalb der letzten drei Dekaden habe sich ein globales politisches System herausgebildet, das eine Vielzahl internationaler Institutionen umfasst und auf staatliches Verhalten einwirkt (Zürn 2018). Daran anknüpfend unternimmt dieser Forumsbeitrag den Versuch, die Gestalt dieses politischen Systems zu umreißen und ihren spezifischen Einfluss auf die internationalen Beziehungen zu skizzieren. Er schlägt vor, Global Governance als ein polyzentrisch strukturiertes System zu

\footnotetext{
${ }^{1}$ Ich danke Kenneth W. Abbott für produktive Gespräche über polyzentrische Regelungsstrukturen.
} 
konzeptualisieren und zu analysieren. Polyzentrisch strukturierte Regelungssysteme bestehen aus vielen Entscheidungszentren, die formal voneinander unabhängig sind, sich aber de facto wechselseitig beeinflussen (V. Ostrom et al. 1961). Polyzentrismus stellt somit die strukturelle Grundlage für Prozesse der Selbstorganisation dar, die soziale Ordnung und die Beilegung von Konflikten auch in Abwesenheit einer hierarchischen Koordinationsinstanz ermoeglichen (V. Ostrom 2014).

Ein polyzentrischer Zugriff auf Global Governance unterscheidet sich von der insbesondere in den 1980er und 1990er Jahren dominierenden monozentrischen Analyseperspektive, die sich anhand von Michael Zürns erstem wegweisenden Beitrag „Interessen und Institutionen in der internationalen Politik“ (Zürn 1992) exemplarisch darstellen lässt. In diesem Buch fragt Zürn nach den Entstehungsbedingungen und der institutionellen Ausgestaltung einzelner internationaler Institutionen, die als separat voneinander operierende Kooperationsprojekte aufgefasst werden (Zürn 1992). Der „frühe Zürn“ steht somit paradigmatisch für den in den 1980er und 1990er Jahren dominierenden analytischen Zugriff auf internationale Institutionen und die im Zentrum der wissenschaftlichen Debatte stehenden Forschungsfragen (Keohane 1984; Hasenclever et al. 1996).

Zürns „Theory of Global Governance“ hingegen nimmt eine systemische Perspektive ein. Sie betont, Global Governance sei mehr als die Summe der Regelungsbemühungen ihrer Einzelinstitutionen, bestehe auch aus Beziehungen zwischen einzelnen Institutionen und deren Einbettung in eine normative Ordnung (Zürn 2018: 6-9). Wiederum steht Zürns Theorie paradigmatisch für den sich seit der Jahrtausendwende zunehmend durchsetzenden Ansatz, internationale Institutionen als eingebettet in Institutionenkomplexe zu konzeptualisieren und zu analysieren (Faude 2015; Gehring/Oberthür 2004; Raustiala/Victor 2004).

Anhand von Michael Zürns zentralen Arbeiten zu internationalen Institutionen lässt sich somit ein Wandel der Analyseperspektive in der theoriegeleiteten Erforschung der Funktionsweise und der Wirkungsbedingungen internationaler Institutionen 
exemplifizieren: Man startete mit einem monozentrischen Zugriff, der im Laufe der Zeit zunehmend von einer polyzentrischen Analyseperspektive abgelöst wurde (Alter/Raustiala 2018). Dieser Wandel der Analyseperspektive vollzieht realweltliche Entwicklungen nach. War das internationale System in den 1980er Jahren relativ schwach institutionalisiert und mit vergleichsweise wenig Autorität ausgestattet, so ist es heute „dicht institutionalisiert“ und mit deutlich mehr Autorität versehen (Zürn 2018: Kapitel 5). Im Ergebnis, so Michael Zuern, sind gegenwärtige internationale Institutionen teil eines globalen politischen Systems, das sich von dem rein anarchisch strukturierten internationalen System unterscheidet, das der monozentrischen Perspektive zugrunde liegt. Allerdings hat die Emergenz eines globalen politischen Systems nicht dazu geführt, dass einzelne internationale Institutionen in eine hierarchische Struktur eingebettet wurden. Auch dem von Michael Zürn identifizierten globalen politischen System fehlt eine durchsetzungsfähige Kollektivinstanz. ${ }^{2}$

Vor diesem Hintergrund unternimmt der vorliegende Forumsbeitrag den Versuch einer Annäherung an die Strukturbedingungen, unter denen sich globale Politik gegenwärtig vollzieht. Im Kern geht es dem Beitrag darum zu skizzieren, wie die polyzentrische Struktur des globalen politischen Systems das Regieren jenseits des Nationalstaats beeinflusst. Zu diesem Zweck geht er folgendermaßen vor: Zunächst wird verdeutlicht, worin die zentralen Merkmale der polyzentrischen Struktur des von Zürn identifizierten globalen politischen Systems bestehen und welche Folgen sich daraus für die Analyse globaler Politik ergeben. Anschließend wird skizziert, wie eine polyzentrische Struktur staatliches Verhalten beeinflusst und das Problem politischer Ordnungsbildung jenseits des Nationalstaates akzentuiert. Schließlich

\footnotetext{
${ }^{2}$ Ein polyzentrisch strukturiertes globales politisches System unterscheidet sich von einem rein anarchischen internationalen System, ist aber nicht notwendigerweise mit der Anarchie-Annahme inkompatibel. Wenn man Anarchie über die Abwesenheit einer zentralen Koordinationsinstanz definiert, dann ist auch ein polyzentrisch strukturiertes System anarchisch. Wenn man Anarchie hingegen über die Abwesenheit jedweder Autoritätsstrukturen definiert, dann geht ein polyzentrisch strukturiertes System über Anarchie hinaus (Donnelly 2015: 410-413).
} 
wird angedeutet, worin die institutionellen Stärken einer polyzentrischen Regelungsstruktur liegen.

\section{Polyzentrismus als analytische Perspektive auf Global Governance}

Das globale politische System des frühen 21. Jahrhunderts ähnelt in seinen Struktureigenschaften den institutionellen Arrangements, die Vincent Ostrom und seine Ko-Autoren in US-amerikanischen Metropolregionen der frühen 1960er Jahre vorgefunden haben (V. Ostrom et al. 1961). Ostrom, Tiebout und Warren beobachteten dort überlappende Zuständigkeiten zwischen einer Vielzahl öffentlicher und privater Institutionen, die nicht durch eine zentrale Koordinationsinstanz gesteuert werden. Sie bezeichneten diese institutionellen Arrangements als polyzentrisch strukturiert.

Eine polyzentrische Struktur definiert sich somit über die Existenz einer Vielzahl von Entscheidungszentren, die formal voneinander unabhängig sind und ein gewisses Maß an Autonomie (sprich: Autorität) besitzen (E. Ostrom 2014: 170). Gleichzeitig sind polyzentrischen Regelungssystemen überlappende Kompetenzbereiche inhärent (Carlislie/Gruby 2017: 7). Ob die einzelnen Entscheidungszentren auch de facto unabhängig voneinander agieren oder wechselseitig aufeinander Bezug nehmen, wird daher als eine empirische Frage angesehen. Sofern sich einzelne Institutionen aufeinander beziehen oder sogar aneinander anpassen, werden sie als Bestandteile eines Regelungssystems gefasst (V. Ostrom et al. 1961: 831-832). Vor diesem Hintergrund kann Polyzentrismus als strukturelle Grundlage eines selbstorganisierenden Systems gelten, in dem soziale Ordnung und die Beilegung von Konflikten auch in Abwesenheit einer hierarchischen Koordinationsinstanz möglich ist (V. Ostrom 2014). Eine wichtige Frage polyzentrischer Forschung ist, wie sich diese strukturellen Bedingungen auf die Produktion von Kollektivgütern auswirken (Carlisle/Gruby 2017).

Analog zu den US-amerikanischen Metropolregionen der frühen 1960er kann das gegenwärtige globale politische System in Anschluss an Michael Zürns „Theory of 
Global Governance" als bestehend aus einer Vielzahl Sektor-spezifischer Institutionen aufgefasst werden, die formal unabhängig voneinander operieren, deren Jurisdiktionen aber teilweise überlappen, ohne in eine formale Hierarchie eingebettet zu sein. Kurzum: das globale politische System des frühen 21. Jahrhunderts weist eine polyzentrische Struktur auf. ${ }^{3}$ Sie ist das nicht intendierte Resultat der Tendenz von Staaten, immer neue Sektor-spezifische internationaler Institutionen $\mathrm{zu}$ gründen und mit internationaler Autorität auszustatten (Alter/Raustiala 2018). Diese Tendenz folgt einer zunehmenden „sozialen und ökonomischen Denationalisierung“, auf die Michael Zürn bereits früh hingewiesen hat (Zürn 1998). Somit sind es in letzter Instanz (welt-)gesellschaftliche Entwicklungen, die komplexere institutionelle Arrangements auf internationaler Ebene und damit ein polyzentrisch strukturiertes globales politisches System hervorgebracht haben (Zürn \& Faude 2013). Durch seine interne Ausdifferenzierung vollzieht das globale politische System die Differenzierung seiner Umwelt nach (Albert et al. 2013: 21). Im Ergebnis vollzieht sich globale Politik nun unter den Bedingungen einer polyzentrischen Struktur. Analog zur Polyzentrismus-Forschung beschäftigt sich daher auch die gegenwärtige Forschung zu internationalen Institutionen mit der Frage, wie diese spezifische institutionelle Struktur die Produktion von Kollektivgütern beeinflusst (Keohane/Victor 2011).

Das polyzentrisch strukturierte globale politische System unterscheidet sich sowohl von einem rein anarchisch strukturierten, als auch von einem hierarchisch strukturierten System. Im Gegensatz zu einem rein anarchisch strukturierten internationalen System besteht die polyzentrische Global Governance aus einer Mehrzahl von internationalen Institutionen, die mit einem Mindestmaß an Autorität ausgestattet sind und kollektiv verbindliche Regelungsstrukturen etablieren

\footnotetext{
${ }^{3}$ Freilich sind US-amerikanische Metropolregionen sowohl in einen Bundes- als auch in einen Nationalstaat eingebettet, während internationalen Institutionen eine vergleichbare Einbettung in weltstaatliche Strukturen abgeht. Allerdings spielt die Einbettung von Metropolregionen in staatliche Strukturen in der Polyzentrismus-Forschung keine Rolle. Forschungsleitend ist vielmehr die Frage, wie es sich auf das Regieren von Metropolregionen auswirkt, wenn die Erfüllung kommunaler Aufgaben nicht in einer hierarchischen, sondern im Rahmen einer polyzentrischen Struktur erfolgt (V. Ostrom et al. 1961: 831). Es ist diese Frage, die sich auf die internationalen Beziehungen übertragen lässt.
} 
(Hooghe et al. 2017). Von einem hierarchisch strukturierten System unterscheidet sich die polyzentrische Global Governance dadurch, dass sie keine hierarchische Koordinationsinstanz enthält, die autoritativ Werte zuweist, das Gewaltmonopol besitzt und Konflikte zwischen Sektor-spezifischen Institutionen autoritativ lösen kann. Kurzum: Dem globalen politischen System fehlt es an systemweiten Autoritätsstrukturen; es ist nicht in eine konstitutionelle Struktur eingebettet (Hurd 2008). Die dem System innewohnende internationale Autorität ist vielmehr institutionen-spezifisch. Sie bezieht sich auf das Verhältnis einer Vielzahl separat gegründeter internationaler Institutionen mit klar umrissenen Regelungsbereichen zu ihren Regelungsadressaten (Zürn 2018: Kapitel 2). Infolge dessen stehen die einzelnen Institutionen des Systems, trotz vielfach überlappenden Kompetenzen, zunächst in einem unkoordinierten Verhältnis zueinander (Faude 2011).

Insgesamt ermöglicht es ein polyzentrischer Zugriff, den durch die Zunahme an institutioneller Dichte und internationaler Autorität veränderten strukturellen Bedingungen globaler Politik Rechnung zu tragen (Alter/Raustiala 2018). Er ist insbesondere für diejenigen attraktiv, die es -- trotz der Tatsache, dass das internationale System weiterhin keine durchsetzungsfähige Kollektivinstanz besitzt -- als nicht erkenntnisfördernd erachten, weiterhin von einem rein anarchisch strukturierten internationalen System auszugehen und eine monozentrische Perspektive auf internationale Institutionen zu werfen (vgl. Donnelly 2015). Gleichwohl war das zentrale Anliegen von Vincent Ostrom und seinen Ko-Autoren, polyzentrische Regelungsstrukturen als eine Alternative $\mathrm{zu}$ hierarchischen Regelungsstrukturen zu etablieren (V. Ostrom et al. 1961: 831). Somit bietet das Konzept des Polyzentrismus einen analytischen Zugriff auf die Strukturbedingungen globaler Politik, der sich einerseits von der theoretischen Annahme eines anarchisch strukturierten international Systems unterscheidet, andererseits aber nicht das Entstehen einer hierarchischen (weltstaatlichen) Struktur postuliert. Es fuehrt somit aus der heuristisch wertvollen, aber analytisch etwas grobkoernigen Dichotomie zwischen anarchisch und hierarchisch strukturierten Regelungssystemen heraus. 


\section{Das Verhalten von Staaten in einer polyzentrischen Struktur}

Die polyzentrische Analyseperspektive etabliert eine neue Makro-Annahme über die Bedingungen, unter denen sich globale Politik vollzieht. Eine solche MakroAnnahme ist allerdings nur dann erkenntnisfördernd, wenn sie plausibel machen kann, dass die von ihr identifizierten strukturelle Bedingungen das Verhalten von Akteuren systematisch in einer Art und Weise beeinflussen, die von bestehenden Makro-Annahmen abweicht. Dies möchte ich ansatzweise versuchen.

Die monozentrische Analyse internationaler Institutionen geht auf Basis der Annahme eines rein anarchisch strukturierten internationalen Systems davon aus, dass Staaten vor dem Hintergrund einzelner und separat voneinander operierender Institutionen bestimmen, ob sie an institutionalisierter Zusammenarbeit in einem Themenbereich partizipieren oder außerhalb dieser institutionellen Struktur operieren (Zürn 1992: Kapitel 2).

Die polyzentrische Analyseperspektive weicht in zweierlei Hinsicht davon ab: Erstens unterstellt sie, dass institutionalisierte Zusammenarbeit für Staaten unabdingbar ist, um wichtige Politikziele $\mathrm{zu}$ erreichen. Die zentrale Hintergrundbedingung einer polyzentrischen Analyseperspektive ist ein hohes Maß an transnationaler Interdependenz oder, anders herum betrachtet, ein geringes Ausmaß an staatlicher Autonomiefähigkeit (Held et al. 1999; Zürn 1998). Letzteres impliziert, dass Staaten nur in geringem Maße in der Lage sind, wichtige Politikziele unilateral zu erreichen. Aus diesem Grund schaffen sie eine Vielzahl internationaler Institutionen, statten diese mit Autorität aus und gewinnen ein Interesse an ihrer dauerhaften Aufrechterhaltung und Funktionsfähigkeit (Hanrieder/Zangl 2015). Denn ein niedriges Maß an staatlicher Autonomiefähigkeit impliziert, dass der unilaterale Rückzug aus institutionalisierter Zusammenarbeit mit hohen Kosten verbunden ist. Somit finden zwischenstaatliche Beziehungen in der polyzentrischen Konstellation unweigerlich innerhalb von internationalen Institutionen statt. ${ }^{4}$

\footnotetext{
${ }^{4}$ Ausgeschlossen ist damit selbstverständlich nicht, dass Staaten aus einzelnen internationalen Institutionen austreten.
} 
Zweitens eröffnet die polyzentrische Struktur Staaten neue Möglichkeiten der institutionellen Wahl (Jupille et al. 2013). Die Verfügbarkeit einer Vielzahl institutioneller Strukturen mit teilweise überlappenden Kompetenzen in Abwesenheit einer zentralen Koordinationsinstanz bietet ihnen die Möglichkeit, ihre Interessen parallel in verschiedenen institutionellen Foren zu verfolgen. Von besonderer Wichtigkeit ist, dass Staaten Möglichkeiten institutionenübergreifenden Handelns gewinnen, die in einem rein anarchisch strukturierten internationalen System nicht zur Verfügung stehen und einer monozentrischen Analyseperspektive deshalb verborgen bleiben (Morse/Keohane 2014). Diese neuen Handlungsmöglichkeiten müssen keineswegs kooperationsfördernd sein, sondern können auch zur Verfolgung parochialer Interessen genutzt werden (Drezner 2013). Denn die zentrale Hintergrundbedingung der polyzentrischen Analyseperspektive unterstellt, dass auch parochiale Interessen nicht mehr unilateral verfolgt werden, sondern in bestehenden oder von unzufriedenen Akteuren neu geschaffenen institutionellen Strukturen (Urpelainen/van de Graaf 2014). Somit wird Unzufriedenheit mit bestehenden institutionellen Strukturen in einem polyzentrisch strukturierten System anders kanalisiert als in einem rein anarchisch strukturierten System, wo sie zu Defektion und unilateralem Handeln führen kann (Morse/Keohane 2014; Zürn 2018: Kapitel 7).

Vor diesem Hintergrund scheint es plausibel, dass die auf einem hohen Ausmaß transnationaler Interdependenz basierende Existenz einer Vielzahl von internationalen Institutionen mit teilweise überlappenden Kompetenzbereichen die Bedingungen, unter denen Staaten auf internationaler Ebene operieren, in einer Art und Weise verändert, die die Grenzen einer mit der Annahme der Anarchie operierenden monozentrischen Analyseperspektive sprengen (vgl. Zürn 1992: Kapitel 2).

\section{Politische Ordnungsbildung in der polyzentrischen Global Governance}

Aus der polyzentrischen Struktur des globalen politischen Systems folgt eine Akzentuierung der Diskussion um politische Ordnungsbildung jenseits des 
Nationalstaates (Zürn et al. 2007). Die monozentrische Analyseperspektive fragt nach den Möglichkeiten politischer Ordnungsbildung innerhalb von einzelnen internationalen Institutionen. Sie konzentriert sich somit auf einzelne „Inseln der Ordnung“ (Strange 1982), verliert dadurch aber die Ordnungsstruktur des Gesamtsystems aus dem Blick (Albert/Cederman 2010: 8-10).

Aus einer polyzentrischen Perspektive stellt sich die Frage nach Möglichkeiten politischer Ordnungsbildung hingegen vor dem Hintergrund überlappender Kompetenzbereiche internationaler Institutionen und Optionen inter-institutionellen Handelns für Staaten. Mit anderen Worten: Eine polyzentrische Perspektive fragt nach den Möglichkeiten von Metagovernance, verstanden als politische Ordnungsbildung zwischen separat voneinander gegründeten internationalen Institutionen (Faude 2015; Holzscheiter 2015). Hier geht es also um die Koordination interdependenter Teilordnungen in Abwesenheit einer hierarchischen Koordinationsinstanz. Die entscheidende Frage ist, ob und wie inter-institutionelle Ordnung in Abwesenheit einer zentralen Koordinationsinstanz möglich ist. Jedenfalls kann erst dann, wenn sich überlappenden Institutionen wechselseitig aufeinander beziehen und inter-institutionelle Konflikte somit auch in Abwesenheit einer hierarchischen Struktur gelöst werden können, von einem integrierten Regelungssystem gesprochen werden (Carlisle/Gruby 2017: 2).

Eine polyzentrische Analyse sucht daher nach inter-institutionellen Ordnungsstrukturen, die als Meta-Institutionen soziale Ordnung zwischen separat voneinander gegründeten Institutionen schaffen. Es handelt sich hierbei um systemische Ordnungsstrukturen globalen Regierens, welche die durch ein Ordnungsprinzip koordinierten Regeln der einzelnen Institutionen enthalten. Sie beruhen auf einer Hierarchie von Ordnungsbeziehungen, innerhalb der die involvierten Ordnungen im Hinblick auf ihr allgemeines Ordnungsprinzip übereinstimmen, sich aber im Hinblick auf konkretere Ordnungsprinzipien unterscheiden. Wenn eine solche Ordnungsstruktur existiert, wirkt sie auf die Handlungsoptionen und Präferenzen der Akteure zurück und beeinflusst somit ihr Verhalten (Faude 2015: Kapitel 4). 
Analog zum Polyzentrismus betont auch der globale Rechtspluralismus das produktive Potential dezentralisierter Regelungsstrukturen und insbesondere die Möglichkeit dezentraler Ordnungsbildung (Krisch 2010: Kapitel 3). Das Konzept des Rechtspluralismus beschreibt eine Situation, in der mindestens zwei Rechtssysteme im selben sozialen Raum koexistieren. Es wurde von Rechtsanthropologen entwickelt, um das Zusammenspiel von normativen Ordnungen in kolonialen und postkolonialen Gesellschaften zu erfassen. Später wurde es auf das internationale Recht übertragen, um dessen Dezentralisierung analytisch zu fassen (Michaels 2009: 5). Eine polyzentrische Analyseperspektive ist somit unmittelbar an den globalen Rechtspluralismus anschlussfähig (Zimmermann et al. 2013).

\section{Die institutionellen Stärken eines polyzentrischen Global Governance Systems}

Vincent Ostrom und seine Ko-Autoren wenden sich gegen die Vorstellung, eine polyzentrische Regelungsstruktur fuehre $\mathrm{zu}$ einer ineffizienten Kollektivgutproduktion in Metropolregionen (V. Ostrom et al. 1961). Sie argumentieren, dass polyzentrische Strukturen effektiver Kollektivgüter produzieren als hierarchische Strukturen, wenn sich Mechanismen der interinstitutionellen Koordination herausbilden (V. Ostrom et al. 1961: 831).

Die Literatur zur Funktions- und Wirkungsweise polyzentrischer Regelungsstrukturen identifiziert drei spezifische institutionelle Stärken, die für die Analyse des globalen politischen Systems instruktiv sein könnten: Aufgrund ihrer dezentralen Struktur sind polyzentrische Regelungsstrukturen erstens besonders gut geeignet, eine komplexe Umwelt zu regieren und zweitens besonders gut in der Lage, sich an eine dynamische Umwelt anzupassen. Drittens reduziert die Verfügbarkeit einer Mehrzahl formal voneinander unabhängiger institutioneller Strukturen das Risiko eines Zusammenbruchs des Gesamtsystems (Carlisle/Gruby 2017: 18-19). Zusammen genommen implizieren diese drei spezifischen institutionellen Stärken, dass sich eine polyzentrische Regelungsstruktur durch ein vergleichsweise hohes Maß an Resilienz auszeichnet. 
Das Konzept der Resilienz wurde traditionell in der Biologie und in der Psychologie verwendet. In der Biologie bezeichnet es die Überlebensfähigkeit einer Spezies unter widrigen Bedingungen und in der Psychologie die Widerstandsfähigkeit von Menschen in herausfordernden Situationen. In jüngerer Vergangenheit hielt das Konzept auch in der Politikwissenschaft Einzug und wurde auf verschiedene Regelungssysteme angewendet (Bhamra et al. 2011; Duit et al. 2010; Folke 2006). Hier bezeichnet es die Widerstandsfähigkeit von Regelungsstrukturen unter schwierigen Umständen in einer sich verändernden Umwelt. Die genauen Definitionen von Resilienz unterscheiden sich leicht, aber alle beziehen sich auf die Fähigkeit von institutionellen Strukturen, produktiv auf veränderte Umweltbedingungen $\mathrm{zu}$ reagieren, die Regelungssysteme herausfordern und politische Antworten erforderlich machen. Angewendet auf internationale Institutionen bezeichnet Resilienz somit deren Fähigkeit, trotz widriger Umweltbedingungen weiterhin zwischenstaatliche Zusammenarbeit $\mathrm{zu}$ ermöglichen.

Auf dieser Basis lässt sich vermuten, dass die polyzentrische Global Governance aufgrund ihrer flexiblen institutionellen Struktur vergleichsweise gut in der Lage ist, mit einer komplexen Umwelt fertig zu werden und zwischenstaatliche Zusammenarbeit auch in stuermischen Zeiten zu ermöglichen (Duit et al. 2010). Die Forschung zu polyzentrischen Regelungssystemen legt nahe, dass insbesondere zwei konkrete Faktoren zur Widerstandsfähigkeit der polyzentrischen Global Governance beitragen: die Diversität und die Redundanz der für die Herstellung zwischenstaatlicher Kooperation zur Verfügung stehenden institutionellen Arrangements (Carlisle/Gruby 2017). Die Diversität institutioneller Arrangements bietet die Möglichkeit kollektiven Handelns auch bei heterogenen Präferenzen von Akteuren. Im Gegensatz zu einer hierarchischen Struktur zwingt eine polyzentrische Struktur Staaten nicht dazu, sich auf eine institutionalisierte Lösung zu einigen, sondern ermöglicht kollektives Handeln in unterschiedlichen institutionellen Kontexten. Die Redundanz institutioneller Arrangements -- also die Verfügbarkeit von institutionellen Strukturen, die ähnliche oder gleiche Regelungsfunktionen erfüllen -- kreiert ein Sicherheitsnetz für Situationen, in denen einzelne Institutionen 
paralysiert sind (Kelley 2009). Angesichts der zunehmenden Umstrittenheit internationaler Kooperation und deren institutioneller Ausgestaltung könnte sich die Fähigkeit polyzentrischer Strukturen, kollektives Handeln auch bei heterogenen Präferenzen zu ermöglichen, als besonders wichtig erweisen. Die Entscheidung des britischen Volkes, aus der Europäischen Union auszutreten, und die Wahl eines Unilateralisten zum Präsidenten der Vereinigten Staaten sind nur die prominentesten Beispiele der neuen Umstrittenheit internationaler Kooperation, deren Ursachen deutlich tiefer liegen als in den schillernden Persönlichkeitsstrukturen einzelner PolitikerInnen.

Betrachtet man mit der internationalen Handelspolitik exemplarisch ein Politikfeld, das für die Entwicklung der monozentrischen Analyseperspektive auf internationale Institutionen besonders wichtig war, so stellt man einerseits fest, dass innerhalb der Welthandelsorganisation (WTO) seit 1995 kein einziger verbindlicher multilateraler Vertrag zwischen allen Mitgliedern abgeschlossen werden konnte. Andererseits fällt auf, dass die Mitglieder der WTO im gleichen Zeitraum 272 präferentiellen Handelsabkommen beigetreten sind (Baccini 2019).

Aus zwei Gründen erscheint es plausibel, beide Entwicklungen auf ein erhöhtes $\mathrm{Ma}$ an Präferenzheterogenität zurückzuführen: zum einen verlagerte sich der inhaltliche Gegenstandsbereich internationaler Handelspolitik von der Reduzierung tarifärer Handelshemmnisse auf die unweigerlich mit größeren Verteilungskonflikten verbundene regulatorische Harmonisierung. Zum anderen stieg die Mitgliedschaft der WTO durch den Beitritt von Entwicklungs- und Schwellenländern seit ihrer Gründung von 123 auf 164 Länder an (Jupille et al. 2013). Im Kern, so lässt sich argumentieren, waren es diese beiden Entwicklungen, die den Abschluss eines verbindlichen Abkommens im Rahmen der WTO unmöglich machten, weil sie zu heterogeneren Praeferenzen zwischen den Mitgliedsstaaten fuehrten. Präferentielle Handelsabkommen hingegen erlauben es, Präferenzheterogenität zu reduzieren.

Die internationale Handelspolitik exemplifiziert daher erstens, dass die monozentrische Analyseperspektive bedeutsame institutionelle Dynamiken nicht 
erfassen kann. Sie ermöglicht es, den Stillstand in der WTO zu analysieren, nicht aber die damit verbundene Proliferation von präferentiellen Handelsabkommen. Um die Entwicklung der internationalen Handelspolitik in den letzten 25 Jahren erklären zu können, ist daher eine polyzentrische Analyseperspektive notwendig. Zweitens exemplifiziert die internationale Handelspolitik, dass polyzentrische Regelungsstrukturen besser als monozentrische Strukturen in der Lage sind, kollektives Handeln bei heterogenen Präferenzen und einer dynamischen Umwelt zu ermöglichen.

Gleichwohl sind polyzentrische Regelungsstrukturen verletzlich. Ihr wunder Punkt polyzentrischer Regelungsstrukturen liegt in der Frage des Zusammenspiels einzelner Institutionen in Abwesenheit einer zentralen Koordinationsinstanz. Denn trotz des Potentials polyzentrischer Regelungsstrukturen, die Resilienz internationaler Kooperation zu staerken, besteht die Moeglichkeit, dass Staaten institutionelle Strukturen errichten, die nicht miteinander kompatibel sind und das Potential polyzentrischer Regelungsstrukturen deshalb nicht realisieren. Vor diesem Hintergrund erlangen Regierungstechniken, die darauf abzielen, die Regelungsbemuehungen internationaler Institutionen mit ueberlappenden Kompetenzbereichen in Einklang zu bringen, besondere Bedeutung (siehe Abbott et al. 2015).

\section{Schlussfolgerungen}

Global Governance bezeichnet globales Regieren in Abwesenheit in einer globalen Regierung (Dingwerth/Pattberg 2006). Insofern unterscheidet sich Global Governance von hierarchisch organisierten politischen Systemen, aber auch von einem rein anarchisch strukturierten System, denen jede Form institutionalisierter Autorität fehlt. Es ergibt sich aus den Regelungstätigkeiten, die unterschiedliche Akteure in vielfältigen institutionellen Strukturen ausführen, ohne dass irgendein Akteur das Gesamtsystem kontrolliert. 
Um ihre Gestalt vor diesem Hintergrund etwas genauer zu umreißen, schlägt dieser Forumsbeitrag vor, Global Governance als polyzentrisch strukturiert aufzufassen. Eine polyzentrische Struktur bezeichnet eine komplexe Form von Governance, die sich innerhalb einer Vielzahl von institutionellen Strukturen vollzieht. Jede dieser Institutionen verfuegt ueber themen-spezifische Autoritaet, überlappt aber auch mit anderen institutionellen Strukturen ohne dass eine hierarchische Koordinationsinstanz für Kohärenz sorgen würde (Carlisle/Gruby 2017: 2). Somit bietet die hier vorgeschlagene polyzentrische Analyseperspektive eine konzeptionelle Annäherung an das, was Michael Zürn bereits im letzten Jahrtausend „Projekt komplexes Weltregieren“ genannt (Zürn 1998) und kürzlich als globales politisches System umschrieben hat (Zürn 2018).

Dieser Beitrag plädiert für eine stärkere Befassung nicht nur mit den Schwächen eines polyzentrisch strukturierten globalen politischen Systems (siehe Hanrieder/Zangl in diesem Forum), sondern auch mit dessen Stärken. Diese liegen in einer vergleichsweise hohen Anpassungsfähigkeit, aber auch in Möglichkeiten zur wechselseitigen Kontrolle internationaler Institutionen und nicht zuletzt in Möglichkeiten der Kontestation bestehender institutioneller Strukturen im Sinne von „contested multilateralism“ (Morse/Keohane 2014). Es gibt somit nicht nur funktionale, sondern auch normative Gründe dafür, die Herausbildung eines polyzentrisch strukturierten globalen politischen Systems als Fortschritt zu begreifen.

\section{Literaturverzeichnis}

Abbott, Kenneth W./Genschel, Philipp/Snidal, Duncan/, Zangl, Bernhard (Hrsg.) 2015: International Organizations as Orchestrators, Cambridge.

Albert, Mathias/Buzan, Barry/Zürn, Michael (Hrsg.) 2013: Bringing Sociology to IR. World Politics as Differentiation Theory, Cambridge. 
Albert, Mathias/Cederman, Lars-Erik 2010: Introduction: Systems Theorizing in IR, in: Albert, Mathias/Cederman, Lars-Erik/Wendt, Alexander (Hrsg.): New Sytems Theories of World Politics, Basingstoke, 3-22.

Alter, Karen/Raustiala, Kal 2018: The Rise of International Regime Complexity, in: Annual of Law and Social Science 14, 18.1-18.21.

Baccini, Leonardo 2019: The Economics and Politics of Preferential Trade Agreements, in: Annual Review of Political Science 22, 75-92.

Bhamra, Ran/Dani, Samir/Burnard, Kevin 2011: Resilience. The Concept, a Literature Review and Future Directions, in: International Journal of Production Research 49: 18: 5375-5393.

Carlisle, Keith/Gruby, Rebecca L. 2017: Polycentric Systems of Governance: A Theoretical Model for the Commons, in: Policy Studies Journal, 1-26.

Dingwerth, Klaus/Pattberg, Philipp 2006: Global Governance as a Perspective on World Politics, in: Global Governance 12: 1, 185-203.

Donnelly, Jack 2015: The Anarchy Discourse in IR, in: International Theory 7: 3, 393-425.

Drezner, Daniel W. 2013: The Tragedy of the Global Institutional Commons, in: Finnemore, Martha/Goldstein, Judith (Hrsg.): Back to Basics. State Power in a Contemporary World, New York, 280-310.

Duit, Andreas/Galaz, Victor/Eckerberg, Katarina/Ebbesson, Jonas 2010: Governance, Complexity, and Resilience, in: Global Environmental Change 20: 3, 363-368. 
Faude, Benjamin 2015: Paradoxe Verrechtlichung: Wie Streitschlichtunsmechanismen interagieren, in Zeitschrift für Internationale Beziehungen 18: 1, 77-108.

Faude, Benjamin 2015: Von Konkurrenz zu Arbeitsteilung. Komplexität und Dynamik im Zusammenspieler internationaler Institutionen, Frankfurt a.M..

Folke, Carl 2006: Resilience. The Emergence of a Perspective for SocialEcological Systems Analyses, in: Global Environmental Change 16: 3, 253-267.

Gehring, Thoma/Oberthür, Sebastian 2004: Exploring Regime Interaction. A Framework for Analysis, in: Underdal, Arild/Young Oran R. (Hrsg.): Regime Consequences. Methodological Challenges and Research Strategies, Dordrecht, 247-269.

Hanrieder, Tine/Zangl, Bernhard 2015: The Embedded State: The New Division of Labor in the Provision of Governance Functions, in: Leibfried, Stephan/Huber, Evelyne/Lange, Matthew/Levy, Jonah D./Stephens, John D. (Hrsg.): The Oxford Handbook of Transformations of the State, Oxford, 250-265.

Hasenclever, Andreas/Mayer, Peter/Rittberger, Volker 1996: Interests, Power, Knowledge: The Study of International Regimes, in: Mershon International Studies Review 40, 177-228.

Held, David/McGrew, Anthony/Goldblatt, David/Perraton, Jonathan 1999: Global Transformations. Politics, Economics and Culture, Palo Alto.

Hooghe, Liesbet/Marks, Gary/Lenz, Tobias/Bezuijen, Jeanine/Ceka, Besir/Derderyan, Svet 2017: Measuring International Authority: A Postfunctionalist Theory of Governance, Volume III, Oxford. 
Holzscheiter, Anna 2015: Interorganisationale Harmonisierung als sine qua non für die Effektivität von Global Governance?, in: da Conceição-Heldt, Eugénia/Koch, Martin/Liese, Andrea (Hrsg.): Internationale Organisationen: Autonomie, Politisierung, interorganisationale Beziehungen und Wandel (Sonderheft 49 der Politischen Vierteljahresschrift), Baden-Baden, 322-348.

Hurd, Ian 2008: After Anarchy. Legitimacy and Power in the United Nations Security Council, Princeton.

Jupille, Joseph/Mattli, Walter/Snidal, Duncan 2013: Institutional Choice and Global Commerce, Cambridge.

Kelley, Judith 2009: The More the Merrier? The Effects of Having Multiple International Election Monitoring Organizations, in: Perspectives on Politics 7: 1, 59-64.

Keohane, Robert O. 1984: After Hegemony. Cooperation and Discord in the World Political Economy, Princeton.

Keohane, Robert O/Victor, David G. 2011: The Regime Complex for Climate Change, in: Perspectives on Politic 11: 1, 7-23.

Krisch, Nico 2010: Beyond Constitutionalism: The Pluralist Structure of Postnational Law, Oxford.

Michaels, Ralf 2009: Global Legal Pluralism, in: Annual Review of Law \& Social Sciences 5: 2, 243-262.

Morse, Julia/Keohane, Robert O. 2014: Contested Multilateralism, in: Review of International Organizations 9: 4, 385-412. 
Ostrom, Elinor 2014: Beyond Markets and States: Polycentric Governance of Complex Economic Systems, in: Ostrom, Elinor/Ostrom, Vincent/Sabetti, Filippo/Aligica, Paul Dragos (Hrsg.): Choice, Rules and Collective Action. The Ostroms on the Study of Institutions and Governance, Colchester, 167-209.

Ostrom, Vincent 2014: Polycentrictiy: The Structural Basis of Self-Governing Systems, in: Ostrom, Elinor/Ostrom, Vincent/Sabetti, Filippo/Aligica, Paul Dragos (Hrsg.): Choice, Rules and Collective Action. The Ostroms on the Study of Institutions and Governance, Colchester, 45-60.

Ostrom, Vincent/Tiebout, Charles M./Warren, Robert 1961: The Organization of Government in Metropolitan Areas: a Theoretical Inquiry, in: American Political Science Review 55: 4, 831-842.

Raustiala, Kal/Victor, David G. 2004: The Regime Complex for Plant Genetic Resources, in: International Organization 58: 2, 277-309.

Strange, Susan 1982: Cave! Hic Dragones: A Critique of Regime Analysis, in: International Organization 36: 2, 479-496.

Urpelainen, Johannes/van de Graaf, Thijs 2014: Your Place or Mine? Institutional Capture and the Creation of Overlapping International Institutions, in: British Journal of Political Science, 1-29.

Zimmermann, Lisbeth/von Staden, Andreas/Marciniak, Angela/Wallbott, Linda/Arndt, Friedrich 2013: Muss Ordnung sein? Zum Umgang mit Konflikten zwischen normativen Ordnungen, in: Zeitschrift für Internationale Beziehungen 20: 1, 35-60.

Zürn, Michael 1992: Interessen und Institutionen in der internationalen Politik: Grundlegung und Anwendung des situationsstrukturellen Ansatzes, Opladen. 
Zürn, Michael 1998: Regieren jenseits des Nationalstaats. Globalisierung und Denationalisierung als Chance, Frankfurt a.M..

Zürn, Michael 2018: A Theory of Global Governance. Authority, Legitimacy \& Contestation, Oxford.

Zürn, Michael/Binder, Martin/Ecker-Ehrhardt, Matthias/Radtke, Katrin 2007: Politische Ordnungsbildung wider Willen, in: Zeitschrift für Internationale Beziehungen 14: 1, 129-164.

Zürn, Michael/Faude, Benjamin (2013): On Fragmentation, Differentiation and Coordination, in: Global Environmental Politics 13: 3, 119-130. 